middle and lower lobes of the right lung are in a state of red hepatisation. The pericardium contains 2 ounces of blood-stained serum. The heart is healthy, but loaded with fat. The valves are all competent and healthy. Abdomen.-The stomach is dilated, and in a state of chronic catil'rh with superficial ulcers. The liver is enlarged and fatty. The spleen is normal. The kidneys are fatty. A large cyst occupies a considerable part of the abdominal cavity. It is firmly adherent anteriorly to the abdominal wall, the adhesions in some parts being cartilaginous in density. The mesentery and bowels are adherent to the cyst. The lymphatic glands, retroperitoneal and mesenteric, are enlarged and indurated. The cyst arises from the fundus of the uterus by a narrow pedicle. The solid part of it is on the right side. The cyst contains a reddish-brown fluid which measures 30 ounces. The entire tumour has been separated from the surrounding parts, and removed along with the uterus and its appendages. Fluid, identical with the contents of the cyst, was found in the peritoneal cavity. The wall of the eyst has undergone fatty degeneration, and posteriorly it is very attenuated; and at the upper and posterior part of the wall, where the thinning is most marked, there is a small perforation about the size of a sixpence. The cyst is unilocular. There is a small cyst about the size of a pigeon's egg in the left ovary.

REMaRKs.- A uterine cystic fibroma is so rare that I need make no apology for bringing this case before the Section. Let me briefly refer to the history of this very remarkable and most interesting case, and, in the first place, the sexual history of the patient and her family deserves to be noted. The patient had a miscarriage at 52 , and did not cease to menstruate until she was 6o. Her mother had a miscarriage at the sixth month when she was 54, and the patient's sister menstruated regularly until she was 54. The patient enjoyed good health up to r870, when she had a miscarriage, which was followed by inflammation. From that time she seems never to have regained.her former health. In June, 1880, ten years later, she had another miscarriage, which was attended with "flooding," and it is to the results of this that she attributes her subsequent troubles. Some months after this miscarriage, she observed that the abdomen was increasing in size, and for a time she thought she was pregnant, but, as the swelling was hard, and did not increase at the same rate and in the same proportions as a gravid uterus, and, moreover, as menstruation was occurring regularly, she suspected that she was the subject of a growth. About that time she consulted Dr. Thomas Keith, who told her that she was suffering from a uterine fibroid tumour. There can be no doubt that the tumour was at first an ordinary fibroid, which, in the course of years, became cystic. In 1885 she was treated with ergot, and under its administration the tumour contracted considerably - a point of some diagnostic value. About this period she was examined by a specialist, who told her that she had an ovarian tumour. It was not until 1887 that the cystic nature of the growth was discovered. It was in October of that year that she was tapped for the first time, when fluid like venous blood was removed from the cyst. A microscropical examination of the fluid was made, when the absence of Drysdale's cells and columnar epithelium helped to confirm the diagnosis. Any slight doubt that may have been previously entertained as to whether the cyst was uterine or ovarian was now removed. Beyond causing her some discomfort, the tumour gave her no further trouble until the month of May, r891, when, owing to extreme tension of the abdominal wall and interference with the respiration and the action of the heart, she had to be relieved by tapping for a second time. The instrument which was used on this as wellas on all subsequent occasions, was Mathieu's aspirator. It is a very remarkable circumstance that there should have been such a long interval as three years and seven months between the first and second tappings. From the second tapping onwards it was found necessary to relieve her, at first at intervals of a few months, and latterly every eight weeks. It was in January, 1892 , at the fourth tapping, that the fluid was for the first time observed to contain pus, which was present on all subsequent occasions. As the pus was sweet I resolved to continue the tappings as long as it remained so, and in the event of its becoming putrid to open the cyst and drain it. From the fifteenth tapping, which was performed in April, 1894, until the twenty-first, which took place in April, 1895, the cyst underwent a steady diminution in size, and the amount of fluid became less at every tapping, falling in the course of the last twelve months from 12 pints to 8 pints. This is an important fact, and it gave me some reason to hope, more especially as the patient was gaining; flesh and strength, that I might eventually effect a cure by emptying the cyst periodically. As to the acute attack of septicæmia, which brought about a fatal issue, I feel certain that it was caused by the perforation of the cyst.

\section{ON THE MENSURATION OF THE DEFORMITY OF HIP DISEASE. ${ }^{1}$}

By A. B. JUDSON, M.D.,

Orthopædic Surgeon to the Out-patient Department of the New York Hospital.

THE object of the present paper is to facilitate methods of recognising, measuring, and recording the degrees of deformity of hip disease. A great advance in the study of this subject was made in 1877 by Mr. Howard Marsh. I reproduce in Figs. I to 5 the original figures with which he illustrated

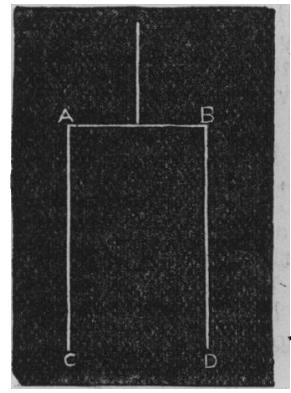

Fig. x.

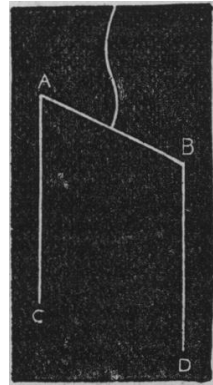

Fig. 3

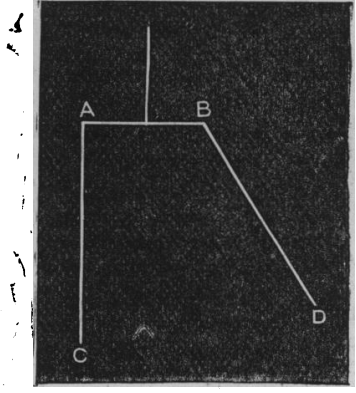

Fig. 2.

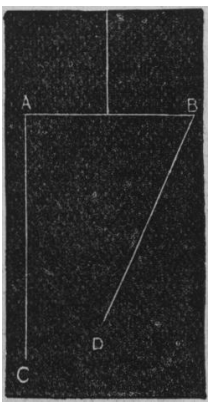

Fig. 4.

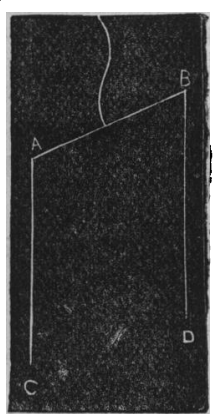

Fig. 5. his first lecture on Hip Disease at the Hospital for Sick Children in London. These figures, modified in various waye, have reappeared many times in the literature of this subject, and to one clinically familiar with these deformities they do not require explanation. Mr. Marsh's lecture contains a complete exposition of the mechanics of the deformity of hip disease, and it will not be easy to add anything to what he has said. ${ }^{2}$ I have, however, constructed the movable models represented in Figs. 6 to 10, in order to make this difficult subject more easy of comprehension.

We appreciate at a glance the clinical significance of abduction or adduction when combined with fixation. Abduction means apparent lengthening, and adduction apparent shortening. Abduction and lengthening, adduction and shortening-flexion and lordosis-are to us mentally interchangeable terms, but it may not have occurred to us all that these factors of deformity may be easily measured and recorded, and that we may thus better appreciate the importance of affording relief and the merits of whatever method we may adopt to remove or prevent deformity. The dolls in Figs. 6 and 7 were arranged and photographed several years ago. They show graphically the degrees of motion and deformity, but they cannot, from the immobility of their vertebral joints, show the effect of the disease on the length of the limb or the curve of the spine. These effects are, however,

1 Presented at the ninth session of the American Orthopædic Association, Clicago, 1895

2 British Medical JOURNAL, July 14 th, 1877, pp. $37-39$ 


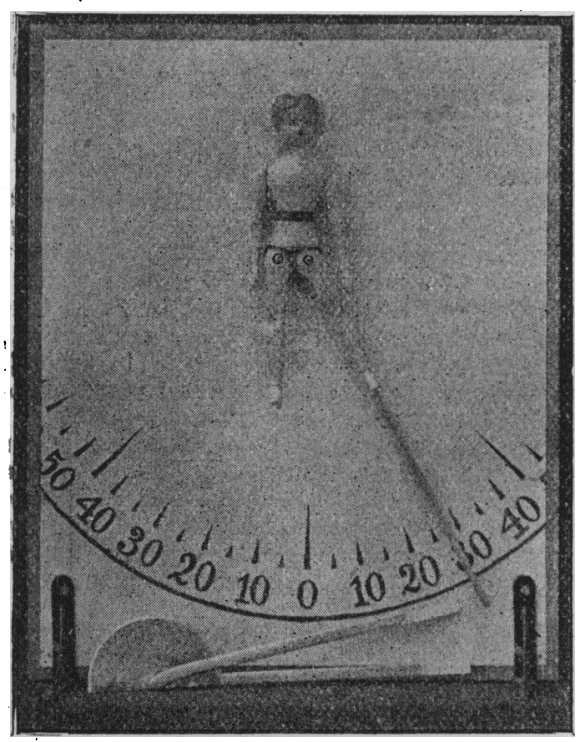

Fig. 6.

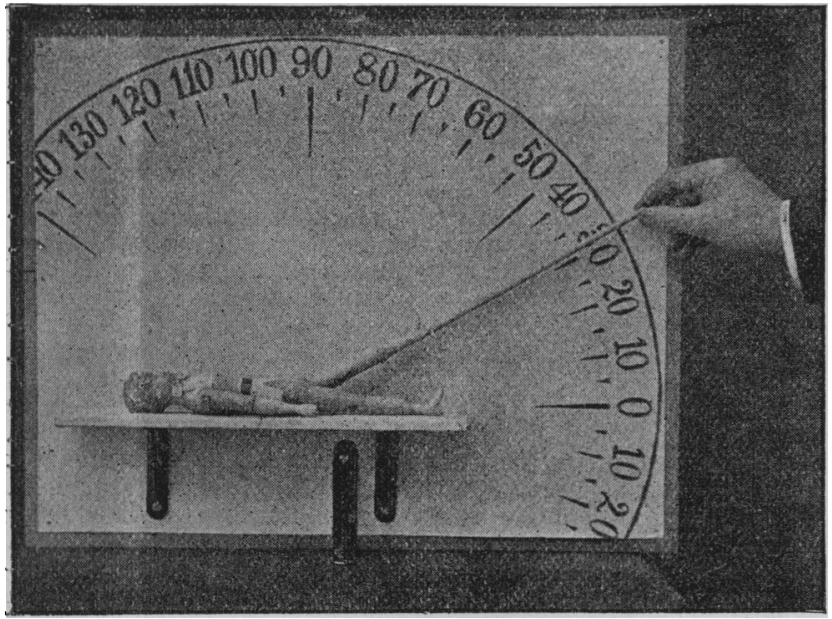

Fig. 7.

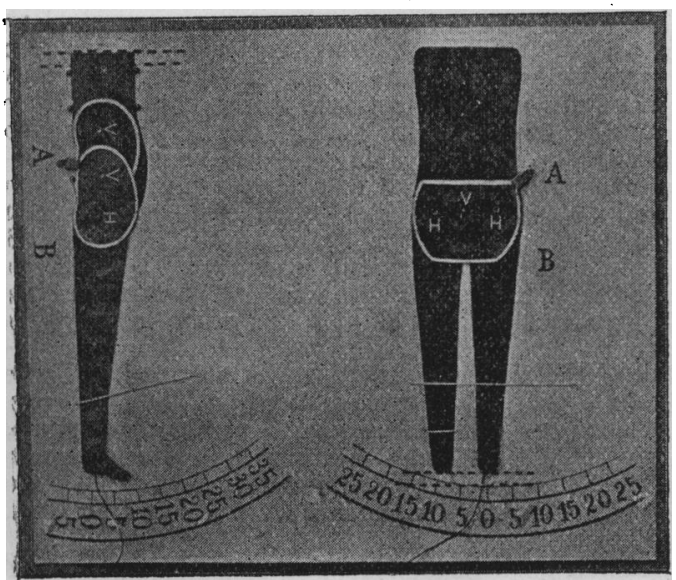

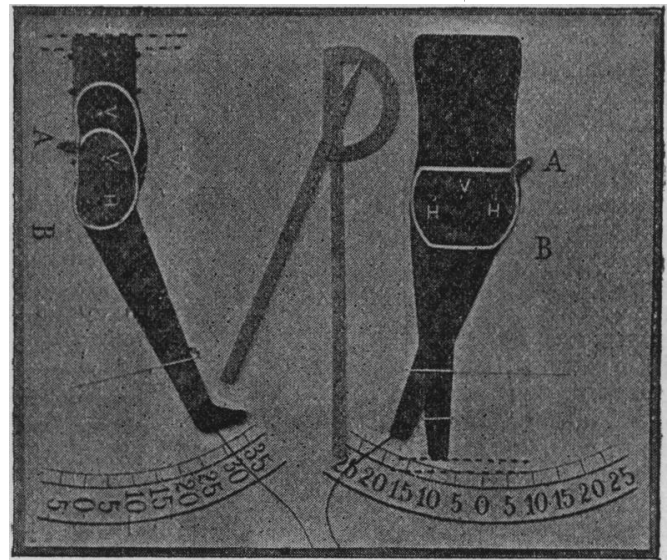

Fig. 9.

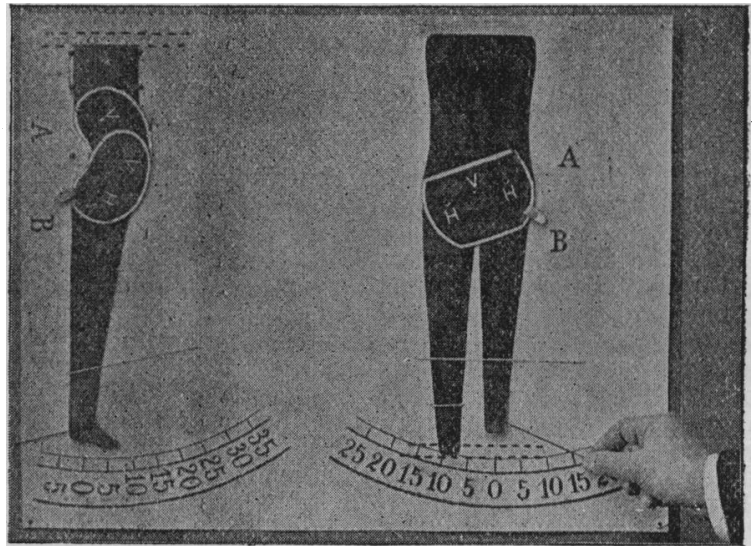

Fig. ro.

shown in Figs. 8 to 10, in which the figures cut out of pressboard take the place of dolls, and are so put together that they exhibit motion and also the arrest of motion, not.only at the hip but also at the vertebral joints.

The figures explain themselves to a certain extent. The joints are made of eyelets not pressed too hard with the "eyelet-set," and loosely screwed to the board at $v$ in the full figure, and at $H$ in the profile. The piece representing the thorax in the profile is reinforced at the back by a thin sheet of brass, as the edges of the press-board were found to wear out against the four screws which act as guides. The spring clip is a "scarf retainer." The apparatus is easily made, and is useful in class demonstration.

To Show the Effect of Lateral Deviation with Fixation.-The full figure is first arranged symmetrically, as in Fig. 8. The clip applied at $\mathrm{A}$ binds together the piece representing the pelvis and that representing the trunk, and thus prevents motion in the vertebral joint, at $\nabla$, while motion at the hipjoint is free. The limb is then drawn into adduction, as in Fig. 9, and the clip is removed from $A$, releasing the vertebral joints, and applied at $B$, where it fixes the hip-joint, at $\mathbf{H}$, by binding together the pieces representing the pelvis and the femur. When an attempt is made to restore symmetry, or to make the limbs parallel, the figure exhibits tilting of the pelvis and apparent shortening, as in Fig. 10. In like manner, abduction and fixation may be made to produce apparent lengthening. A fortunate result is that in which the patient recovers with enough apparent lengthening to annul the real shortening caused by loss of bone or unequal growth.

To Show the Effect of Flexion with Fixation.-The cuts may be turned sideways so as to present the profile recumbent. The clip applied at $A$; as in Fig. 8, binds together the pieces 
representing the pelvis and the trunk, and thus prevents motion in the vertebral joints, $v \mathrm{v}$, while motion at the hip, at $\mathbf{H}$, is free. The limb is then raised in flexion, as in Fig. 9, and the clip is moved from $A$ to $B$, releasing the vertebral joints and fixing the hip-joint. When an attempt is then made to reduce flexion or lower the limb to the table the result is lordosis.

It is interesting to note, by observing the transverse dotted lines, that the patient's height is increased by either adduction or abduction, and decreased by flexion when either is combined with fixation. The height is diminished in lateral curvature of the spine by the sigmoid curve, in Pott's disease by kyphosis, and in hip disease by lordosîs.

The deformity of hip disease may readily, and with sufficient accuracy, be measured by the use of the goniometer, two forms of which are shown in Figs. 6 and 9. This instrument .s simple in construction, and may well be in frequent clinical use. The one shown in Fig. 9 is made by attaching arms to a protractor.

To Measure Adduction or Abduction.-The limb is slowly moved in the direction of adduction and abduction alternately until, after repeated trials, it is seen that when it is held at a certain point in the arc of lateral motion the iliac spines are at right angles with the axis of the trunk. One arm of the goniometer is then held parallel "with a line connecting the iliad spines. If the table is against the wall of the room, it is convenient to see that the iliac spines are at right angles with the wall, and then the arm of the goniometer may be directed point blank at the wall. The other arm is then made to coincide with a line parallel, as near as may be, with the axis' of the limh, which may, for this purpose, be considered to extend from the middle of Poupart's ligament to the middle of the heel. If the axis of the thigh only is considered, the incidental presence of genu valgum or varum would impair the value of the observation. The degrees may then be counted on the scale of the protractor.

To Measure Flexion.- With one hand the limb is slowly raised and lowered alternately until, after repeated trials, it is found that when the limb is held at a certain point the lumbar spines press gently on the fingers of the other hand placed under the back. One arm-of the goniometer is then held horizontally by the hand released from under the back, while the other arm is made to coincide, as near as may be, with a line parallel with the axis of the mass of the limb. The degrees then read off on the inner scale of the protractor are the degrees of flexion. Although its determinations are, from the nature of the case, only approximate, the goniometer is an instrument of precision, and as such finds a place in the consulting room.

Writers on this subject have usually viewed deformity as the result of immobilisation of the hip-joint. The figures and models referred to in the preceding pages have been constructed with that view. As a matter of fact, however, few cases present absolutely immovable joints at any stage. There is almost always considerable motion. In the acute stage the joint, which at the first glance appears to be motionless, is found to yield through an are of many degrees in response to gentle force rightly applied. Later in the course of the disease many cases show wide and free passive motion in different directions, and the point at which motion is arrested often varies from day to day; and even in after-life the position of the limb is subject to considerable variation. The use of the goniometer facilitates recognition of these phenomena. Further observation in this direction may add to our knowledge and to the efficiency of treatment.

\section{THE TREATMENT OF LEUCOCYTHAMIA WITH BONE MARROW.}

By J. R. WHAIT, M.B., B.Sc., M.R.C.S., South Hampstead.

THE following case may present some features of sufficient interest to justify publication.

A lady, aged 56, came under observation on September 2oth, 1894, complaining of pain in the left side, enlargement of abdomen, dyspnoea, cough, weakness, and loss of flesh. There was nothing important in her history except that during the menopause and since (menstruation ceased in 1891 at age of 53) she had a good deal of mental worry, to which she attributed her illness.

In January, 1894, she found that she was getting thinner, and in June or July that her abdomen was getting large and uncomfortable.

The patient was thin, but not at all anæmic-looking. The tongue was coated, the pulse 100 , and the temperature $99.4^{\circ}$. The spleen was enlarged and extended downwards to $\mathrm{I} \frac{1}{2}$ inch above the iliac crest, and inwards to a spot $3 \frac{1}{2}$ inches to the left of the middle line. It was firm but not tender. The liver reached 2 inches below the costal margin. The lymphatic glands were not enlarged. On the inner aspect of the left tibia the bone was thickened and very tender. There was no sign or history of hæmorrhage. The blood contained a large excess of leucocytes: red corpuscles numbered 4, 150,000 per c.mm.; the leucocytes 329,360 per c.mm., a proportion of 1 to 12.6 red cells. The hæmoglobin was 60 per cent. of normal. There were numbers of poikilocytes and granule masses present.. She was ordered liq. arsenicalis m $v$ three times a day, and the dose increased by $m$ ij every third day.

On September 27th the spleen was considerably larger. The blood remained unaltered.

On October 6th her condition was about the same as on September 27th, and she was then taking liq. arsenicalis m xij thrice daily.

Remembering the success with which Professor Fraser had treated a case of pernicious anæmia with bone marrow, as reported in the BRITISH MEDICAL Journal of the previous. June, I resolved to try it in this case. Dr. Robert Maguire, who kindly saw the patient with me at this time, concurred; and she was put under the treatment at once. It consisted in the administration of a dessertspoonful of ox bone marrow spread on toast three times a day with meals. The arsenic was continued as before. She began to improve almost immediately.

On October gth the spleen was slightly smaller. Red corpusoles, 4,050,000 per c.mm.; leucocytes, 190,000 per c.mm., or 1 to 21.3 red cells. The arsenic was producing gastric irritation, and the dose had to be diminished $m \mathrm{x}$.

On October 26th the spleen was still smaller. Red corpuscles, 3,510,000 per c.mm.; leucocytes, 46,000 per c.mm. or. 1 to 76.3 red cells. Very few tailed red cells were left.

On November roth both spleen and liver were greatly reduced ; the red corpuscles had risen to $4,170,000$ per c:mm. the leucocytes fallen to 25,000 per c.mm., or i to 167 red. Ferri carb. sacchar. gr. xx, ti.d., was added to the treatment.

On December 8th the red corpuscles were 4,200;000 per c.mm, leucocytes, 20,000 per c.mm., or 1 to 210 red cells. The hæmoglobin had risen to 70 per cent. By this time, however, marked symptoms' of peripheral neuritis had appeared. The tongue was very white; the skin was dry and taking on a brownish hue. The hands and feet were suffused, tender, and benumbed. She was unable to perform the finer coordinative movements, and there was considerable jerking of the limbs. The prick of a pin anywhere on the soles of the feet or on the toes was imperfectly felt and localised. There was also tinnitus and deafness. The arsenic was again reduced, but the symptoms got stadily worse. The bronzing of the skin getting so marked as to suggest Addison's disease. It was universal, except for a small white patch the size of a threepenny piece over the upper end of each sacro-iliac joint. The pigmentation was exaggerated at the nipples, axilla, umbilicus, etc. Speech became blurred, tonguedry and brown, and the urine scanty and loaded with urates. There was great desire for sleep; for several days the patient slept twenty-two hours out of the twenty-four.

On December 24th the arsenic was stopped. The spleen still filled the whole left hypochondrium. The red corpuscles were 4,300;000 per c.mm.; leucocytes, 19,500 per c.mm., or I to 220 red cells. The fresh marrow had become very distasteful to the patient, and ' $I$ substituted for it tabloids of compressed marrow. Of these 6 were given daily, and later increased to. 9 .

The neuritis getting worse, on December 28th hypodermic injections of strychnine were commenced ( $\frac{1}{60}$ gr. t.i.d.), and by January 4 th she could control the jerkings and her grasp 